\title{
Determinação de parâmetros cinéticos da sinterização de revestimentos cerâmicos de monoqueima do tipo BIIa
}

\author{
(Determination of kinetic parameters of sintering of \\ ceramic type BIIa single firing)
}

\author{
M. Cargnin ${ }^{1,2}$, S. M.A. G.U.de Souza ${ }^{1}$,A.A.U.de Souzal ${ }^{1}$ A. De Noni Jr., \\ ${ }^{\prime}$ Programa de Pós-Graduação em Engenharia Química, Universidade Federal de Santa Catarina, \\ Florianópolis, SC 88040-900 \\ ${ }^{2}$ Instituto Maximiliano Gaidzinski, R. Edson Gaidzinski 352, Cocal do Sul, SC 88845-000 \\ ${ }^{3}$ Universidade do Extremo Sul Catarinense, Av. Universitária 1105, Bairro Universitário, Criciúma, SC 88806-000
}

\begin{abstract}
Resumo
A produção de revestimentos cerâmicos pelo processo monoqueima é atualmente o mais difundido entre as empresas de cerâmica. O processo de queima está em constante evolução e adaptação à novos parâmetros, devido ao crescimento tecnológico e à diversificação dos produtos cerâmicos. É necessário entender o mecanismos envolvidos na queima de placas cerâmicas utilizando fornos a rolos, principalmente o desempenho térmico do equipamento e características técnicas dos revestimentos cerâmicos. Neste trabalho, um modelo matemático e um procedimento experimental foram aplicados para determinar a cinética de retração. Os parâmetros cinéticos foram determinados experimentalmente, utilizando pó industrial para obter corpos-de-prova de $80 \mathrm{x} 20 \mathrm{x}$ $2,3 \mathrm{~mm}^{3}$ queimados em forno elétrico laboratorial a rolo. A energia de ativação, $\mathrm{E}_{\mathrm{a}}=178041 \mathrm{~J} / \mathrm{mol}$, o expoente do tempo " $n$ " que determina o mecanismo de sinterização, $n=0,26285$ e fator de freqüência, $\mathrm{k}_{0}=44768 \mathrm{~s}^{-1}$ foram determinados depois de distintas condições de queima em 30, 40 e 50 min de frio a frio e as temperaturas entre 1000 a $1150{ }^{\circ} \mathrm{C}$ ( $\operatorname{com}$ passos de $30{ }^{\circ} \mathrm{C}$ ).
\end{abstract}

Palavras-chave: queima, sinterização, cinética, revestimentos cerâmicos.

\begin{abstract}
The firing process is the core of the tiles production, currently the single-cycle firing is the most widespread method in ceramic industries. Moreover, the increasing demand of technological improvements and technical diversifications of ceramic products, leads the firing process to continuously adapt to new operative conditions. The physical mechanisms that occur in roller kilns have to be understood, mainly those involving thermal performance of the equipment and technical characteristics of the ceramic tiles. In this work, a mathematical model and an experimental procedure has been applied to determine the shrinkage kinetics. The kinetic parameters have been experimentally determined using industrial powder to obtain test pieces of $80 \times 20 \times 2.3 \mathrm{~mm}^{3}$ fired in laboratory electrical roller kiln. Activation energy, $E_{a}=178 \mathrm{~kJ} / \mathrm{mol}$, parameter " $n$ ", $n=0.26285$ and frequency factor, $k_{0}=44768 \mathrm{~s}^{-1}$ were determined after several firing conditions 30,40 and 50 min cold-to-cold and temperatures from 1000 to $1150{ }^{\circ} \mathrm{C}$ (steps of $30^{\circ} \mathrm{C}$ ).

Keywords: firing, sintering, kinetic, ceramic tile.
\end{abstract}

\section{INTRODUÇÃO}

O processo de produção de placas cerâmicas para revestimentos por monoqueima é, atualmente, o mais difundido entre as indústrias cerâmicas em todo o mundo. Este processo de produção através de fornos a rolos vem evoluindo através dos tempos, seja pelo aumento das dimensões dos equipamentos, maior eficiência energética, maior produtividade por unidade área ocupada e ao uso do gás natural. A etapa de queima (tratamento térmico) é determinante na obtenção das propriedades desejadas ao produto final, pois será transferida a energia térmica necessária para promover uma série de reações químicas e físicas como, por exemplo, decomposições térmicas, transformações alotrópicas, formação de fase líquida, sinterização entre outras $[2,5,11]$. Com isso a estabilidade nesta etapa deve ser mantida, tanto na alimentação das placas cerâmicas para o interior do forno como na manutenção da curva e ciclo de queima ao longo de todo o equipamento.

A maior preocupação dos fabricantes de revestimentos cerâmicos, além das características estéticas, é a de garantir a obtenção de produtos dentro de uma mesma categoria dimensional (tamanho), pois muito embora a grande parte dos problemas de perda de qualidade não seja originada na operação de queima, é nesta etapa que se busca a resolução dos problemas no produto. Esta estabilidade está relacionada diretamente com a temperatura e tempo de queima, além do tipo de produto a ser fabricado. O controle da retração linear é amplamente utilizado no controle do tamanho do produto final, pois os requisitos de qualidade das placas 
cerâmicas para revestimentos são estabelecidos através de normas nacionais (NBR 13818) e internacionais (ISO 13006), sendo que um destes requisitos é a uniformidade dimensional. O tamanho final de uma placa cerâmica é o resultado das variações dimensionais sofridas durante o processo produtivo, desde a conformação até a queima.

A temperatura de queima é um dos fatores determinantes para a obtenção das propriedades técnicas dos revestimentos cerâmicos. A variação transversal de temperatura no forno é responsável por desvios dimensionais nos revestimentos cerâmicos. Os fatores ligados diretamente ao equipamento são a má regulagem entre a proporção ar/gás, o que provoca oscilações na qualidade da combustão e como consequiência variações de temperatura, o tipo de queimador utilizado (baixa ou alta velocidade), má regulagem na curva de queima, entre outros. Um dos pontos preocupantes na queima em fornos a rolos consiste na redução do gradiente de temperatura na direção transversal do forno [4]. A existência destes gradientes, com maiores temperaturas no centro e menores nas laterais, causa problemas dimensionais nas placas cerâmicas produzidas, com maiores retrações na parte de maior temperatura, gerando diferentes lotes de produto na etapa de classificação. Além da influência da temperatura, o tempo de queima também afeta as características das placas cerâmicas, principalmente, em ciclos rápidos, pois a troca de calor entre o ambiente e a peça faz com que a superfície seja aquecida mais rapidamente que o seu interior, dando origem a gradientes de temperatura [3]. Portanto, variações de temperatura podem interferir diretamente sobre a qualidade do produto final. Adicionalmente às necessidades de controle de variação dimensional, as indústrias buscam de forma constante o aumento da produtividade através da redução dos ciclos de queima. Para isso, busca-se o desenvolvimento de massas mais fundentes que, para uma mesma temperatura e um menor tempo de queima, desenvolvam as mesmas propriedades técnicas, principalmente, tamanho e absorção de água. Tradicionalmente os diagramas de gresificação constituem a ferramenta técnica mais empregada para estudos desta natureza e resultam em informações de orientação quanto a possibilidade de atingir os objetivos de otimização. Por outro lado, a sinterização com presença de fase líquida é o principal fenômeno que provoca a redução da porosidade, retração de queima e desenvolvimento das propriedades nos revestimentos cerâmicos. Este fenômeno pode ser descrito através de modelos cinéticos que aportam informações mais relevantes do ponto de vista termodinâmico. Os modelos cinéticos ainda podem ser empregados juntamente com modelagem e simulação de processos para uma otimização mais rápida, robusta e minimizando os esforços laboratoriais e industriais.

Modelos cinéticos foram utilizados, em estudos laboratoriais, para avaliar os mecanismos de sinterização de diferentes tipos de materiais cerâmicos. $O$ efeito da temperatura e do tempo de queima na densificação de peças cerâmicas de baixa porosidade (pavimento para monoqueima) foi avaliado através de um modelo cinético relacionado com a redução da porosidade do material [6].
Da mesma forma, esta propriedade foi avaliada para uma massa de porcelanato contendo nefelina sienita. O efeito provocado pela adição de nefelina sienita através de um modelo baseado na retração linear de queima foi estudado [9]. Este tipo de estudo também foi realizado para avaliar os mecanismos de sinterização de cerâmicas avançadas $[1,13]$.

O objetivo do presente trabalho é a obtenção do modelo cinético de sinterização de uma massa empregada na produção de revestimentos cerâmicos de monoqueima do tipo BIIa. Para isso verificou-se a necessidade do desenvolvimento de uma metodologia experimental simples e rápida que possa ser empregada de forma geral para qualquer tipo de massa destinada a fabricação desta classe de produtos.

Alguns dos parâmetros mais importantes que provocam as variações dimensionais são a composição química e mineralógica das matérias-primas e razão de mistura deles, distribuição granulométrica, temperatura do corpo cerâmico, $d L$ é a diferença entre o tempo de queima [9], sendo estes dois últimos os parâmetros avaliados neste trabalho. A variação dimensional das placas cerâmicas, que ocorre durante o processo de queima, pode ser obtida a partir da retração linear $\left(R_{l}\right)$ sofrida pela placa. Este valor pode ser calculado tendo-se as dimensões iniciais $\left(L_{0}\right)$, ou seja, as dimensões antes do processo de queima e finais $\left(L_{f}\right)$, através da Eq. A.

$$
\mathrm{R}_{1}=\left(\mathrm{L}_{0}-\mathrm{L}_{\mathrm{f}}\right) / \mathrm{L}_{0}
$$

A variação da retração linear durante o processo de queima pode ser descrita por uma equação similar à Eq. B $[1,9]$ :

$$
\mathrm{R}_{1}=\mathrm{dL} / \mathrm{L}_{0}=\mathrm{k} \cdot \mathrm{t}^{\mathrm{n}}
$$

onde $R_{l}$ é a retração da dimensão inicial $L_{0}$ e a final $L_{f} t$ é o tempo de sinterização, " $k$ " é uma constante da taxa e $n$ é um parâmetro que depende do mecanismo de sinterização dominante. A constante $k$ ' pode ser correlacionada com a temperatura pela Eq. C do tipo Arrhenius:

$$
\mathrm{k}^{\prime}=\mathrm{k}_{0} \cdot \exp \left(-\mathrm{E}_{\mathrm{A}} / \mathrm{R} \cdot \mathrm{T}\right)
$$

onde $k_{0}$ é um fator de frequiência, $E_{A}$ é a energia de ativação, $R$ é a constante do gás ideal e $T$ é a temperatura do corpo cerâmico.

Para obter a equação que descreve a cinética de sinterização da placa cerâmica através da retração linear em função da temperatura $\mathrm{T}$ e tempo $\mathrm{t}$ é necessário substituir a Eq. C em D, utilizando os valores de retração como uma característica do processo de queima, portanto:

$$
\mathrm{R}_{1}=\mathrm{k}_{0} \cdot \exp \left(-\mathrm{E}_{\mathrm{A}} / \mathrm{R} \cdot \mathrm{T}\right) \cdot \mathrm{t}_{\mathrm{n}}
$$

\section{PROCEDIMENTO EXPERIMENTAL}

O modelo cinético apresentado neste artigo [9] utiliza uma equação do tipo Arrhenius. Nesta equação, os valores 
dos parâmetros " $n$ ", " $E_{A}$ " e " $k_{0}$ " dependem do tipo de material e do processo de queima. Desta forma, é necessário encontrar os valores destes parâmetros para o tipo de massa que compõe o revestimento cerâmico que está sendo estudado.

Para a avaliação da cinética de sinterização é necessário verificar o efeito da temperatura máxima de queima e do tempo total de queima na retração linear. Desta forma, buscou-se adotar um procedimento experimental próximo aquele normalmente utilizado para elaborar corpos-de-prova para caracterização técnica. Assim, foram conformados corpos-de-prova pelo processo de prensagem uniaxial a seco, utilizando uma prensa hidráulica laboratorial. A pressão específica utilizada foi de $25 \mathrm{MPa}$. As dimensões dos corposde-prova eram de $80 \mathrm{~mm} \times 20 \mathrm{~mm}$ e espessura $2,3 \mathrm{~mm}$. A massa de pó (7,0 g cada) foi pesada em uma balança digital Micronal com resolução $0,01 \mathrm{~g}$. A secagem das amostras ocorreu em uma estufa laboratorial Fanem 315 SE com temperatura entre $110 \pm 10{ }^{\circ} \mathrm{C}$ até a completa retirada da umidade. As dimensões dos corpos-de-prova secos foram medidas com o uso de um paquímetro digital Mitutoyo com resolução $0,01 \mathrm{~mm}$.

A condição de espessura reduzida foi utilizada para minimizar o efeito da espessura da placa cerâmica na retração linear durante o processo de queima. Com isso, a variação da temperatura entre a superfície e centro da placa cerâmica é minimizada, pois há uma menor quantidade de material a ser aquecido. Assim o efeito da resistência condutiva do calor é minimizado, proporcionando uma homogeneidade na temperatura e conseqüentemente maior uniformidade nas características técnicas da placa cerâmica. As placas cerâmicas produzidas industrialmente, assim como os corpos-de-prova obtidos em laboratório, normalmente apresentam espessuras maiores $(5-8 \mathrm{~mm})$ que provocam gradientes de temperatura entre a superfície e o centro da mesma. Em consequiência, afetam o resultado final da queima e interferem na determinação da relação tempo $\mathrm{x}$ temperatura $\mathrm{x}$ sinterização.

A massa específica a seco das amostras foi obtida através do método de empuxo de mercúrio, para verificar se todas as amostras obtiveram o mesmo grau de compactação, diminuindo assim, o efeito desta variável na retração linear entre os corpos-de-prova.

A queima ocorreu em forno laboratorial a rolos da Sere SRL e modelo ERL 12. A câmara de queima possui $1235 \mathrm{~mm}$ de comprimento por $200 \mathrm{~mm}$ de largura. As temperaturas máximas de queima foram de 1000, 1030, 1060, 1090, 1120 e $1150{ }^{\circ} \mathrm{C}$ com 30,40 e 50 min de tempo total de queima (frio a frio). Para cada condição de tempo e temperatura foram utilizados três corpos-de-prova, totalizando 54 amostras, sendo inseridos no forno sobre placas refratárias, deixando as amostras espaçadas $25 \mathrm{~cm}$ umas das outras para evitar oscilação na temperatura da atmosfera de queima e conseqüente variação na retração linear.

Através dos dados de temperatura, tempo e retração linear é possível determinar os valores dos parâmetros " $n$ ", " $E_{A}$ " e " $k_{0}$ ” para avaliar a cinética de sinterização das placas cerâmicas deste trabalho.
O método utilizado para a determinação dos parâmetros “ $n$ " e " $E_{A}$ " estão baseados em métodos reportados $[1,7,11]$. Para a obtenção destes parâmetros foi necessário linearizar a Eq. D que corresponde ao modelo cinético apresentado [1, 7 , 9]. Desta forma, foi possível obter uma relação linear entre o logaritmo natural da retração $\left(\ln R_{l}\right)$ com o logaritmo natural do tempo $(\ln t)$ para cada temperatura máxima. $\mathrm{O}$ coeficiente angular de cada equação linear obtida é o valor do parâmetro " $n$ ", sendo que para este trabalho foi adotado o valor da média aritmética do parâmetro.

A energia de ativação $\left(E_{A}\right)$, das diferentes condições, foi obtida através da relação linear entre o logaritmo natural da retração $\left(\ln R_{l}\right)$ com o inverso da temperatura máxima em Kelvin $(1 / T)$. Da mesma forma, o coeficiente angular de cada equação linear é o valor da energia de ativação, sendo que para este parâmetro também foi adotado o valor da média aritmética. O último parâmetro a ser determinado foi o valor de " $k_{0}$ ", sendo que nas referências utilizadas, não está clara a forma de determinação deste parâmetro. Existe um efeito de compensação ou isocinético entre os parâmetros cinéticos, sendo que alterações na energia de ativação provocam mudanças no valor do fator pré-exponencial " $k_{0}$ " [9]. A obtenção de " $k_{0}$ " foi feita através de uma aproximação estatística utilizando o desvio percentual (Eq. E) entre os dados da retração linear experimental e os dados calculados pelo modelo cinético. O desvio-padrão foi determinado pela Eq. F.

$$
\begin{aligned}
& \mathrm{D}_{\mathrm{v}}(\%)=\left(\frac{\mathrm{R}_{\text {lcal }}-\mathrm{R}_{\text {lexp }}}{\mathrm{R}_{\text {lexp }}}\right) \times 100 \\
& \mathrm{R}_{\mathrm{MS}}(\%)=\sqrt{\sum_{\mathrm{i}=1}^{\mathrm{n}} \frac{\mathrm{D}_{\mathrm{v}}{ }^{2}}{\mathrm{n}}}
\end{aligned}
$$

A fim de verificar, se os valores encontrados para os parâmetros cinéticos " $n$ ", " $E_{A}$ " e " $k_{0}$ " representam o comportamento da retração linear das placas cerâmicas durante a operação de queima, foram elaboradas correlações entre a retração linear experimental e a retração linear obtida pelo modelo cinético $[8,9]$.

\section{RESULTADOS E DISCUSSÃO}

As placas cerâmicas obtidas pelo processo de prensagem possuem densidade aparente média de $1847 \mathrm{~kg} / \mathrm{m}^{3} \mathrm{com}$ um desvio-padrão de $\pm 3 \mathrm{~kg} / \mathrm{m}^{3}$, confirmando a uniformidade do processo de compactação e minimizando o efeito desta propriedade na retração linear de queima. Adeterminação dos parâmetros cinéticos deu-se através da obtenção da retração linear de queima das placas cerâmicas não esmaltadas em função de diferentes condições de temperatura e tempo de queima. A Fig. 1 apresenta às curvas de queima utilizadas neste trabalho com um tempo de queima de frio-a-frio de $30 \mathrm{~min}$. As curvas de queima para os tempos de 40 e 50 min são análogas (mesmo tempo de patamar, porém com 


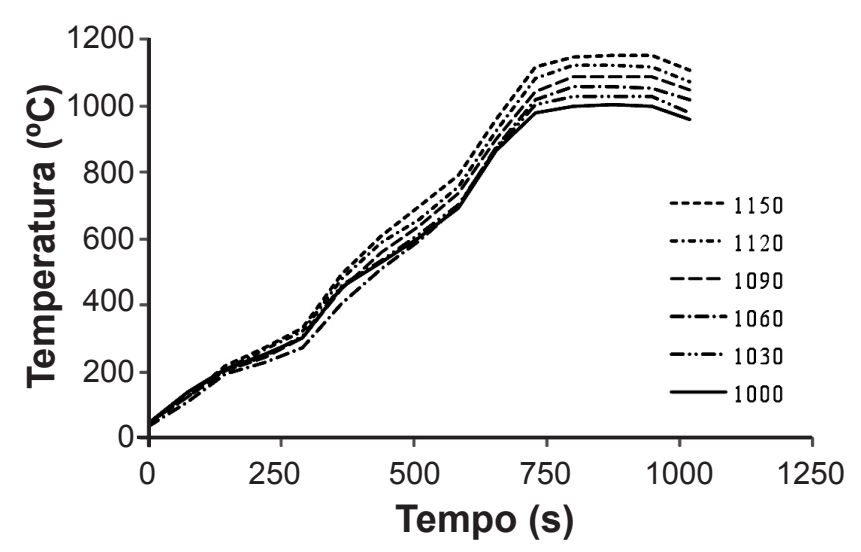

Figura 1: Curvas de aquecimento e patamar de queima para ciclo total de $30 \mathrm{~min}$ (frio-a-frio).

[Figure 1: Heating and soaking time curves for 30 min of firing time (cold-to-cold).]

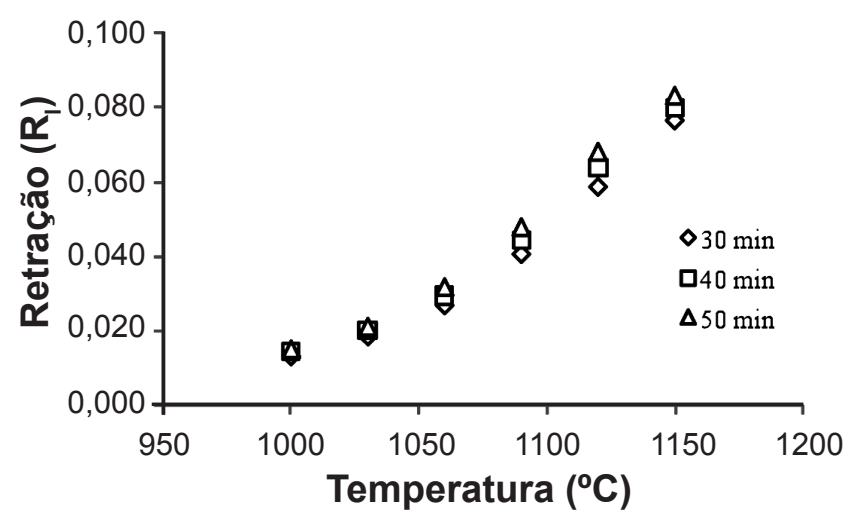

Figura 2: Dados experimentais de retração linear em função do tempo de queima.

[Figure 2: Experimental data of linear shrinkage as a function of firing time.]

menores taxas de aquecimento). A Fig. 2 apresenta o comportamento da retração linear em função da temperatura e tempo de queima. Pode-se perceber que em temperaturas mais baixas $\left(1000{ }^{\circ} \mathrm{C}\right.$ e $\left.1030{ }^{\circ} \mathrm{C}\right)$, não se observa grande influência do tempo de queima. Esta pequena retração devese à decomposição dos materiais argilosos e o mecanismo de sinterização atuante até a temperatura de $1000{ }^{\circ} \mathrm{C}$ é a difusão superficial, onde pequenas retrações são obtidas [12]. para argilas iliticas-cloriticas pequenas retrações acontecem em temperaturas menores que $900{ }^{\circ} \mathrm{C}$, e no intervalo entre 950 a $1000{ }^{\circ} \mathrm{C}$, ocorre um aumento significativo da retração linear, onde o mecanismo atuante é através do fluxo viscoso [14]. Em temperaturas mais elevadas $\left(1060{ }^{\circ} \mathrm{C}, 1090{ }^{\circ} \mathrm{C} \mathrm{e}\right.$ $1120^{\circ} \mathrm{C}$ ), esta variação é maior, mostrando que a influência do tempo é mais significativa. Nesta fase da sinterização ocorre um aumento na quantidade de fase líquida presente no material, devido à quantidade de compostos alcalinos e alcalino-terrosos ( $\mathrm{Na}, \mathrm{K}$ e $\mathrm{Mg}$ ), promovendo um aumento na densificação do corpo-de-prova e conseqüentemente uma maior retração linear [12]. Na fase final da sinterização, as placas cerâmicas tendem a atingir o ponto de máxima densificação em temperaturas acima de $1150{ }^{\circ} \mathrm{C}$, para este caso, a taxa de retração linear é reduzida e o tempo passa a ter uma contribuição pouco menos importante, porém não desprezível. O modelo cinético citado por diversos autores e representado pela Eq. D, requer a determinação de alguns parâmetros, a fim de obter os valores de retração linear dos materiais cerâmicos para determinar os parâmetros cinéticos é necessário linearizar a Eq. D, conforme demonstrado pela Eq. G $[1,7]$.

$$
\ln \left(R_{1}\right)=\ln \left[k_{0} \cdot \exp \left(-E_{A} / R \cdot T\right) \cdot t^{n}\right]
$$

Reorganizando a Eq. G na forma de uma equação linear, tem-se:

$$
\ln \left(\mathrm{R}_{\mathrm{l}}\right)=\ln \mathrm{k}_{0}-\mathrm{E}_{\mathrm{A}} / \mathrm{R} \cdot \mathrm{T}+\mathrm{n} \cdot \ln \mathrm{t}
$$

Através desta linearização (Eq. H), pode-se fazer uma relação entre o logaritmo natural da retração linear de queima $\left(\ln R_{l}\right)$ com o logaritmo natural do tempo $(\ln t)$ para as diferentes temperaturas máximas, conforme demonstrado na Fig. 3. O coeficiente angular das equações lineares é o valor do parâmetro cinético " $n$ " para cada temperatura máxima e estão apresentados na Tabela I. Pode-se perceber uma pequena variação nestes valores para as temperaturas até $1120{ }^{\circ} \mathrm{C}$. Pode-se relacionar esta variação à taxa de retração linear constante, mas para temperaturas iguais e superiores a $1150{ }^{\circ} \mathrm{C}$, ocorre uma redução na taxa de retração e conseqüentemente uma mudança no valor do parâmetro "n". Os coeficientes de correlação linear demonstram que a relação é bem significativa, portanto o valor utilizado para o modelo cinético da retração linear pode ser obtido através da média aritmética, sendo igual a 0,26285 [1, 7,9]. A energia de ativação $E_{A}$ foi determinada através da relação entre o logaritmo natural da retração linear $\left(\ln R_{l}\right)$ e o inverso da temperatura absoluta de queima $1 / T$. O coeficiente angular desta relação é o termo $\left(-\mathrm{E}_{\mathrm{A}} / \mathrm{R}\right)$, sendo $R$ a constante dos gases ideais. A inclinação das equações não é afetada, de maneira significativa, pelo tempo total de queima, conforme apresentado pela Fig. 4. Os valores encontrados para a energia de ativação em cada tempo de queima estão

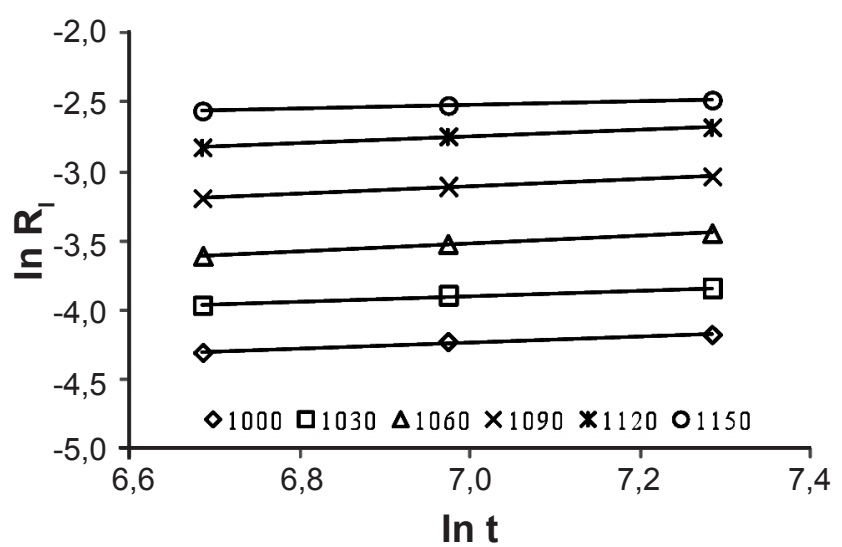

Figura 3: Determinação do valor do expoente do tempo (n). [Figure 3: Determination of the time exponent (n) value.] 
Tabela I - Valores obtidos para o expoente do tempo (n). [Table I - Values for the time exponent (n).]

\begin{tabular}{ccc}
\hline Temperatura $\left({ }^{\circ} \mathrm{C}\right)$ & Expoente do tempo $(\mathrm{n})$ & $\mathrm{R}^{2}$ \\
\hline 1000 & 0,2611 & 0,9978 \\
1030 & 0,2516 & 0,9969 \\
1060 & 0,3185 & 0,9976 \\
1090 & 0,3110 & 0,9984 \\
1120 & 0,2820 & 0,9995 \\
1150 & 0,1529 & 0,9982 \\
$\mathrm{n}_{\text {médio }}=$ & 0,2629 & \\
\hline
\end{tabular}

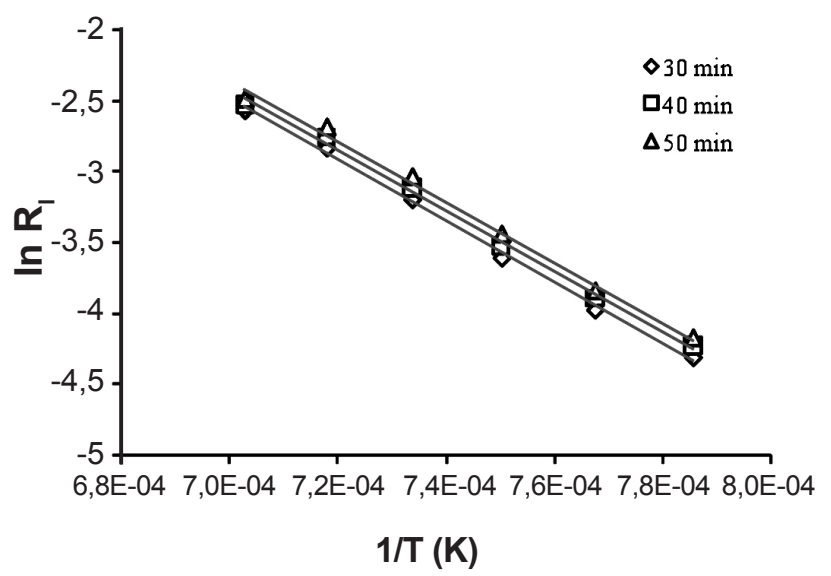

Figura 4: Determinação da energia de ativação $\left(\mathrm{E}_{\mathrm{A}}\right)$.

[Figure 4: Determination of the activation energy $\left(E_{A}\right)$.]

Tabela II - Valores obtidos para a energia de ativação $\left(\mathrm{E}_{\mathrm{A}}\right)$. [Table II - Values for the activation energy $\left(E_{A}\right)$.]

\begin{tabular}{ccc}
\hline $\begin{array}{c}\text { Tempo de Queima } \\
(\mathrm{min})\end{array}$ & $\begin{array}{c}\text { Energia de Ativação - } \mathrm{E}_{\mathrm{A}} \\
(\mathrm{J} / \mathrm{mol})\end{array}$ & $\mathrm{R}^{2}$ \\
\hline 30 & 179665 & 0,9976 \\
40 & 177248 & 0,9965 \\
50 & 176908 & 0,9946 \\
$\mathrm{E}_{\text {a médio }}=$ & 178041 & \\
\hline
\end{tabular}

apresentados na Tabela II. Outro fator que influencia nos valores da energia de ativação é a composição química da massa cerâmica.

A energia de ativação está diretamente relacionada com a quantidade de sódio $(\mathrm{Na})$ e potássio $(\mathrm{K})$ existente nas massas cerâmicas $[9,12]$. Quanto maior é esta quantidade, maiores são os valores para a energia de ativação e conseqüentemente maiores valores de retração linear. A placa cerâmica em estudo é composta por matérias-primas que apresentam quantidades significativas de sódio $(\mathrm{Na})$ e potássio $(\mathrm{K})$, resultando em 4,39\% destes álcalis. Portanto, o valor médio obtido para a energia de ativação é de $178 \mathrm{~kJ} / \mathrm{mol}$ sendo maior que $163 \mathrm{~kJ} / \mathrm{mol}$ [6], pois a quantidade de álcalis é da ordem de $3,93 \%$ [15]. O fator de freqüência $k_{0}$ foi determinado utilizando uma aproximação estatística a partir dos valores encontrados para o parâmetro $n$ e a energia de ativação $E_{A}$. Os valores obtidos estão apresentados na Tabela III e podese perceber uma grande similaridade entre estes valores. Desta forma, o valor utilizado no modelo cinético será a média aritmética, igual a $44768 \mathrm{~s}^{-1}$. Os valores encontrados para os parâmetros cinéticos $n, E_{A}$ e $k_{0}$ foram aplicados na equação do modelo cinético proposto $[8,9]$. Os resultados de retração linear obtidos pelo modelo foram comparados com os valores experimentais, conforme demonstrado pela Fig. 5. Verifica-se que os dados apresentam um alto índice de correlação linear $\left(\mathrm{R}^{2}=0,991\right)$, ficando evidente que os parâmetros obtidos atendem à cinética de sinterização até temperaturas de $1150{ }^{\circ} \mathrm{C}$. Torna-se importante ressaltar que para temperaturas elevadas, acima de $1150{ }^{\circ} \mathrm{C}$, pode ocorrer o surgimento de outros efeitos ou mecanismos de sinterização não contemplados pelo modelo estudado.

Tabela III - Valores obtidos para o fator de frequiência $\left(\mathrm{k}_{0}\right)$. [Table III - Values for the frequency factor $\left(k_{0}\right)$.]

\begin{tabular}{cc}
\hline $\begin{array}{c}\text { Tempo de Queima } \\
(\mathrm{min})\end{array}$ & $\begin{array}{c}\text { Fator de freqüiencia }-\mathrm{k}_{0} \\
\left(\mathrm{~s}^{-1}\right)\end{array}$ \\
\hline 30 & 44811 \\
40 & 44751 \\
50 & 44742 \\
$\mathrm{k}_{\text {0médio }}=$ & 44768 \\
\hline
\end{tabular}

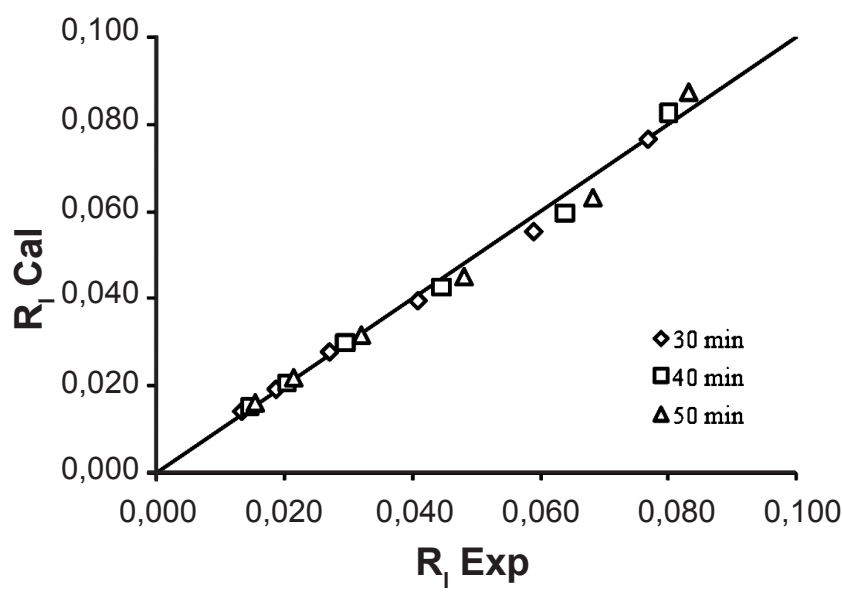

Figura 5: Comparação entre os valores experimentais e os calculados pelo modelo cinético. Correlação de $\mathrm{R}^{2}=0,991$.

[Figure 5: Comparison between experimental values and those calculated by the kinetic model. Correlation $R^{2}=0.991$.]

\section{CONCLUSÕES}

Os parâmetros cinéticos do modelo proposto por Salem et al. [9] foram determinados utilizando dados experimentais de retração linear, temperatura e tempo de queima empregando-se para isso um forno a rolo laboratorial e peças com espessura reduzidas $(2,3 \mathrm{~mm})$. Estas condições experimentais e o cuidado em manter os corpos de prova com a mesma densidade aparente a seco foram fundamentais 
para a qualidade dos dados gerados. O método utilizado para a determinação dos parâmetros cinéticos foi a realização de ajustes lineares da equação do modelo cinético. Estes parâmetros foram obtidos para placas cerâmicas sem a camada decorativa. Os valores obtidos para os parâmetros cinéticos do modelo para retração linear demonstram que a temperatura de queima tem uma importância significativa em seus valores. Para o parâmetro " $n$ " é verificado que para a temperatura de $1150{ }^{\circ} \mathrm{C}$ e acima desta, outro mecanismo está atuando sobre a sinterização do material, pois seu valor $\mathrm{n}_{1150{ }^{\circ} \mathrm{C}}=0,1529$ difere aproximadamente $46 \%$ dos demais valores. A energia de ativação apresentou valores muito próximos para diferentes tempos de queima, tendo como valor médio de $178 \mathrm{~kJ} / \mathrm{mol}$, demonstrando que esta variável não afeta significativamente no valor deste parâmetro. $\mathrm{O}$ fator de freqüência determinado para cada tempo de queima através de um ajuste estatístico entre a retração linear experimental, o parâmetro cinético $n$ e a energia de ativação, apresentou valores muito próximos para cada condição. Portanto, o tempo de queima não contribui para mudanças apreciáveis neste parâmetro cinético. Os resultados obtidos de retração linear a partir do modelo cinético demonstram uma boa concordância com os dados experimentais obtidos em laboratório, tornando possível utilizar esta ferramenta para avaliar o comportamento de uma massa cerâmica para revestimentos de monoqueima do tipo BIIa. Dada a intervalo de validade do modelo ele também pode ser aplicado para revestimentos do tipo BIIb. Os resultados mostraram ainda que este trabalho é capaz de auxiliar no desenvolvimento de curvas de queima apropriadas para cada tipo de situação, ou seja, alterando as condições de tempo e temperatura máxima de queima.

\section{AGRADECIMENTOS}

À empresa Eliane S.A. Revestimentos Cerâmicos pela disponibilidade de suas instalações e ao Instituto Maximiliano Gaidzinski pelo suporte financeiro para a realização deste trabalho.

\section{REFERÊNCIAS}

[1] E. R. Benavidez, C. J. R. González Oliver, Anais Jornadas SAM (2001) 859-866.

[2] W. D. Callister Jr., Ciência e Engenharia de Materiais: Uma introdução, LTC, Rio de Janeiro, RJ (2002).

[3] C. Ferrer, D. Llorens, G. Mallol, E. Monfort, A. Moreno, Técnica Cerámica 227 (1994) 653-662.

[4] T. G. Jahn, Levantamento de dados experimentais e simulação térmica de um forno a rolos a gás natural, Diss. Mestrado, Depto. Eng. Mecânica, UFSC, Florianópolis, SC (2007).

[5] W. D. Kingery, H. K Bowen, D. R. Uhlmann, Introduction to ceramics, John Wiley \& Sons, Cambridge, EUA (1975).

[6] M. J. Orts, J. L. Amorós, A. Escardino, A. Gozalbo, C. Feliu, Appl. Clay Sci. 8, 2-3 (1993) 231-245.

[7] B. B. Panigrahi, M. M. Godkhindi, K. Das, P. G. Mukunda, P. Ramakrishnan, Mater. Sci. Eng. A 396, 1-2 (2005) 255-262.

[8] B. B. Panigrahi, M. M. Godkhindi, Intermetallics 14, 2 (2006) 130-135.

[9] A. Salem, S. H. Jazayeri, E. Rastelli, G. Timellini, J. Mater. Proc. Technol. 209, 3 (2009) 1240-1246.

[10] A. Salem, S. H. Jazayeri, E. Rastelli, G. Timellini, Thermochim. Acta 503-504 (2010) 1-7.

[11] L. E. Vieira, Avaliação do comportamento térmico de uma placa cerâmica ao longo do processo de monoqueima em forno a rolos, Diss. Mestrado, Depto. Ciência e Eng. Materiais, UFSC, Florianópolis, SC (2002).

[12] C. Zanelli, M. Raimondo, M. Dondi, G. Guarini, P. M. T. Cavalcante, Recopil. Qualicer 1990-2006 1 (2004) 257270.

[13] W. Zeng, L. Gao, L. Gui, J. Guo, Ceram. Int. 25, 8 (1999) 723-726.

[14] M. Hajjaji, A. Khalfaoui, S. Kacim, J. Eur. Ceram. Soc. 26 (2006) 161-167.

[15] M. J. Orts, A. Escardino, J. L. Amorós, F. Negre, Appl. Clay Sci. 8, 2-3 (1993) 193-205.

(Rec. 22/10/2010, Ac. 19/05/2011) 